\title{
PRODUÇÃO DE SUBSTRATOS SERS EFICIENTES ATRAVÉS DA DEPOSIÇÃO DE OURO SOBRE UM MOLDE DE MICROESFERAS DE POLIESTIRENO
}

\author{
Diego Pereira dos Santos, Gustavo Fernandes Souza Andrade, ${ }^{\S}$ Antônio Carlos Sant’Ana ${ }^{\S}$ e Marcia L. A. Temperini* \\ Departamento de Química Fundamental, Instituto de Química, Universidade de São Paulo, CP 26077, 05513-970 São Paulo - SP, Brasil
}

Recebido em 22/6/10; aceito em 15/9/10; publicado na web em 25/10/10

\begin{abstract}
PRODUCTION OF EFFICIENT SERS SUBSTRATES BY DEPOSITING GOLD OVER A POLYSTYRENE BEADS TEMPLATE. This work reports on the SERS activity of a nanostructured substrate that was obtained by electrodepositing gold over a template consisting of polystyrene microspheres. This substrate displayed superior SERS performance for the detection of 4-merctaptopyridine as compared to a conventional roughened Au electrode. In order to investigate the substrate capability for the detection at low concentration limits, a series of Rhodamine $6 \mathrm{G}(1 \mathrm{nM})$ spectra were registered. Our spectral dynamics data is in agreement with single-molecule behavior, showing that the control over the substrate morphology is crucial to enable the production of highly reproducible and sensitive SERS substrates.
\end{abstract}

Keywords: SERS; nanostructured Au substrate; single-molecule detection.

\section{INTRODUÇÃO}

O efeito Raman consiste no espalhamento inelástico da radiação eletromagnética incidente, de tal forma que a diferença entre as energias das radiações incidente e espalhada inelasticamente consiste em um quantum vibracional. ${ }^{1}$ Pela origem do fenômeno ser muito diferente da espectroscopia de absorção no infravermelho (IR), devem ser obedecidas regras de seleção diferentes (variação da polarizabilidade molecular com a vibração e não do momento de dipolo como na espectroscopia IR). Apesar das inúmeras vantagens que a espectroscopia Raman proporciona para o estudo das vibrações moleculares, como maior facilidade no preparo de amostras, possibilidade de se trabalhar em meio aquoso, etc., a técnica apresenta baixa secção de choque se comparada à espectroscopia IR, tornando difícil sua aplicação na detecção de espécies moleculares em baixas concentrações. A secção de choque da espectroscopia Raman é cerca de 8 ordens de magnitude menor que a do IR, apresentando valores típicos de $10^{-30} \mathrm{~cm}^{2}{ }^{2}$

O efeito SERS (Surface-Enhanced Raman Scattering) consiste na intensificação do espalhamento Raman de espécies adsorvidas em determinadas superfícies metálicas com formatos e tamanhos bem definidos (escala nanométrica), ${ }^{3}$ de modo que para determinadas condições se podem atingir 10 ordens de grandeza em intensificação da secção de choque Raman. ${ }^{4}$ Este efeito de intensificação levou a espectroscopia Raman para o seleto grupo das espectroscopias com capacidade de detectar espécies no regime de uma única molécula. ${ }^{5,6}$

Dois modelos são normalmente utilizados para explicar a origem desta intensificação. O mecanismo químico leva em consideração a formação de novos estados de energia com a adsorção da molécula na superfície metálica, o que leva a alterações na polarizabilidade molecular e, portanto, do momento de dipolo induzido pela radiação incidente, o que é de extrema importância para o efeito Raman. ${ }^{7,8}$

O modelo eletromagnético leva em consideração a resposta óptica das nanopartículas metálicas em relação ao campo elétrico da radiação

*e-mail: mlatempe@iq.usp.br

"Este trabalho é dedicado ao Prof. Dr. Hans Viertler por ocasião do seu $70^{\circ}$ aniversário.

${ }^{\S}$ Endereço permanente: Instituto de Ciências Exatas, Universidade Federal de Juiz de Fora excitante. A resposta óptica de metais como $\mathrm{Au}, \mathrm{Ag}$ e $\mathrm{Cu}$ pode ser descrita com grande eficácia (principalmente para Ag) pelo modelo de elétrons livres de Drude, em que a polarizabilidade é descrita segundo o modelo de Lorentz, que considera o campo excitante como uma perturbação que leva a polarizabilidade a se comportar como um oscilador harmônico clássico amortecido. ${ }^{9}$ Neste contexto, existe uma condição de ressonância com a frequência da radiação excitante que depende do tipo de metal e dos parâmetros geométricos do mesmo. Esta ressonância é conhecida como ressonância de plasmon de superfície e ocorre na região do visível do espectro eletromagnético para partículas metálicas de dimensões de $10-100 \mathrm{~nm} .{ }^{10}$

A ressonância de plasmon de superfície leva à intensificação do campo local na superfície metálica e, consequentemente, do sinal Raman das moléculas próximas à superfície, que depende da intensidade da radiação incidente. Esta intensificação do campo elétrico localmente à superfície é o fator mais importante na intensificação do sinal Raman pelo efeito SERS., 2,8,11

Uma vez que a ressonância de plasmon de superfície e a intensificação de campo provocada dependem da geometria das nanopartículas, bem como a interação entre as mesmas dada pelo distanciamento entre partículas, ${ }^{12}$ técnicas litográficas são de grande importância para a obtenção de substratos SERS-ativos, ou seja, superfícies metálicas que permitem a intensificação do efeito Raman. ${ }^{13}$

Uma vantagem evidente deste procedimento é o controle sobre o processo de síntese que permite duas características de fundamental importância para o efeito: obtenção de geometrias que possibilitem as maiores intensificações SERS e, controle sobre a reprodutibilidade dos espectros SERS obtidos nestas superfícies.

As duas características apontadas são essenciais para o estudo tanto dos aspectos fundamentais do efeito SERS como também da sua aplicação nos mais variados campos do conhecimento. Desta forma, muitas pesquisas são realizadas na busca de substratos SERS-ativos que apresentem as características mencionadas. Diversos procedimentos são realizados para a obtenção destes substratos, como deposição de filmes metálicos sobre microesferas ${ }^{14}$ ou a utilização de moldes para deposição controlada de partículas metálicas como membranas de policarbonato. ${ }^{15}$ Neste trabalho pretendeu-se explorar a utilização de microesferas de poliestireno depositadas uniformemente sobre uma superfície de $\mathrm{Au}$, como molde para crescimento controlado de estruturas de Au eletrodepositadas. ${ }^{16}$ Com estes substratos espera-se 
a promoção eficiente do efeito SERS na detecção de espécies moleculares em baixa concentração.

\section{PARTE EXPERIMENTAL}

Lâminas de vidro previamente limpas com solução piranha foram tratadas com solução de 3-mercaptopropiltrimetoxissilano (Aldrich, 98\%) $5 \%$ em volume em n-hexano (Synth, P.A.) segundo procedimento reportado na literatura. ${ }^{17} \mathrm{~A}$ solução piranha consiste em solução $2 \%$ em volume de $\mathrm{H}_{2} \mathrm{O}_{2}$ (Synth) saturada em $\mathrm{H}_{2} \mathrm{SO}_{4} 98 \%$ m (Synth); esta solução é extremamente oxidante e deve ser manuseada com extremo cuidado. Após este procedimento de silanização, foi depositada sobre a superfície de vidro modificada uma camada de ouro com espessura de $40 \mathrm{~nm}$, por bombardeamento por plasma de $\mathrm{Ar}^{+}$dirigido sobre um alvo de Au, utilizando-se o equipamento Edwards Scancoat Six, no qual a espessura da camada de Au depositada é controlada através de uma microbalança de quartzo Edwards modelo FTM6.

A superfície metálica foi tratada com solução etanólica de hidrocloreto de cisteamina, $1,0 \times 10^{-2} \mathrm{~mol} \mathrm{~L}^{-1}$, por $48 \mathrm{~h}$. O tratamento com hidrocloreto de cisteamina tem por objetivo modificar a superfície de $\mathrm{Au}$, tornando mais eficiente a adsorção de microesferas de poliestireno (PS) através de interação eletrostática entre PS (normalmente carregado negativamente, devido à presença de grupos sulfônicos para estabilização destas microesferas) e a superfície metálica modificada com a cisteamina na forma protonada no grupo amínico.

A superfície modificada foi então imersa em uma dispersão (1\%) de microesferas de PS de diâmetro $500 \mathrm{~nm}$ em água (Alfa Aesar). A velocidade de emersão dos substratos dessa dispersão foi controlada por um motor de passos interfaceado a um computador. ${ }^{18} \mathrm{~A}$ velocidade de emersão foi controlada a $1,8 \mu \mathrm{m} \mathrm{s}^{-1}$ para deposição uniforme de PS sobre a superfície metálica.

Estas superfícies foram utilizadas como eletrodo de trabalho em uma cela eletroquímica, tendo como eletrodos de referência e auxiliar, $\mathrm{Ag}|\mathrm{AgCl}| \mathrm{KCl}_{\text {(sat) }}$ e um fio de platina, respectivamente. Efetuou-se eletrodeposição nesta superfície de Au através de uma solução de ácido tetracloroáurico ( $\left.\mathrm{HAuCl}_{4}, 12 \mathrm{~g} \mathrm{~L}^{-1}\right)$, EDTA $\left(5 \mathrm{~g} \mathrm{~L}^{-1}\right), \mathrm{Na}_{2} \mathrm{SO}_{3}$ $\left(160 \mathrm{~g} \mathrm{~L}^{-1}\right), \mathrm{K}_{2} \mathrm{HPO}_{4}\left(30 \mathrm{~g} \mathrm{~L}^{-1}\right) .{ }^{19}$ Para a eletrodeposição foi aplicada uma densidade de corrente de $1,0 \mathrm{~mA} \mathrm{~cm}^{-2}$ por $630 \mathrm{~s}$, após o qual as microesferas de PS foram removidas por imersão do substrato em tetra-hidrofurano por $5 \mathrm{~min}$. Após este procedimento, o substrato foi exaustivamente lavado com água deionizada para a sua utilização como substrato SERS-ativo. O substrato assim obtido foi denominado substrato de vidro-Au.

A morfologia do substrato vidro-Au obtido foi avaliada através de microscopia eletrônica de varredura (SEM) e microscopia de força atômica (AFM). As imagens SEM foram obtidas no Laboratório Nacional de Luz Síncrotron (LNLS) e na central analítica do Instituto de Química da Universidade de São Paulo em equipamento JSM-7401F, Field Emission Gun Scanning Electron Microscope. As imagens AFM foram obtidas no Instituto de Física da Universidade de São Paulo em um microscópio SPM NanoScope IIIA, Digital Intruments.

O desempenho do substrato vidro-Au na obtenção de espectros SERS foi comparado com o de um eletrodo ativado por ciclos de oxidação e redução (ORC) preparado segundo procedimento apresentado na literatura. ${ }^{20}$ Um eletrodo de Au $99,99 \%$ de diâmetro $0,13 \mathrm{~cm}^{2}$ previamente polido foi submetido a 25 ciclos de oxidação e redução, em solução aquosa de $\mathrm{KCl} 0,1 \mathrm{~mol} \mathrm{~L}^{-1}$, no intervalo de potenciais de $-0,3$ a $1,3 \mathrm{~V}$, com velocidade de varredura de $100 \mathrm{mV} \mathrm{s}^{-1}$. O intervalo de potenciais foi ajustado para se obter valores máximos de densidade de corrente anódica no intervalo de $10-13 \mathrm{~mA} \mathrm{~cm}^{-2}$. As substâncias utilizadas na investigação do desempenho SERS dos substratos foram 4-mercaptopiridina (4-pyS, obtida da Aldrich, 99\%) e rodamina 6G (R6G, obtida da LambdaChrome, Inc). Para a R6G, foi preparada uma solução-mãe com concentração de $1,0 \times 10^{-4} \mathrm{~mol} \mathrm{~L}^{-1}$, e foram feitas diluições a partir dela; para alcançar a concentração de $1 \times 10^{-9} \mathrm{~mol}$ $\mathrm{L}^{-1}$ foram realizadas duas diluições, primeiro para $1 \times 10^{-7} \mathrm{~mol} \mathrm{~L}^{-1}$, seguida pela diluição para a concentração final desejada.

Os espectros SERS foram obtidos em um equipamento Renishaw Raman System 3000 acoplado a um microscópio metalúrgico Olympus BH2-UMA, utilizando radiação excitante de $632,8 \mathrm{~nm}$ de um laser de He-Ne Coherent 31-2140-000. Os espectros SERS foram obtidos na geometria de retroespalhamento, com excitação e coleção a $180^{\circ}$ uma da outra, e tanto a excitação e a coleção da radiação espalhada foram realizadas pela mesma lente objetiva. A objetiva utilizada possui aumento de 50x com distância de trabalho de $8 \mathrm{~mm}$ e abertura numérica (NA) de 0,55 ; a objetiva não foi imersa na solução de trabalho em momento algum. As medidas SERS foram realizadas com o analito (4-pyS ou R6G) presente na cela eletroquímica na concentração de estudo durante a aplicação de potencial e obtenção dos espectros.

\section{RESULTADOS E DISCUSSÃO}

A Figura 1 apresenta uma imagem SEM do substrato de Au confeccionado segundo o procedimento descrito na Parte Experimental. Uma imagem SEM para maiores ampliações pode ser encontrada na Figura 1S, material suplementar.

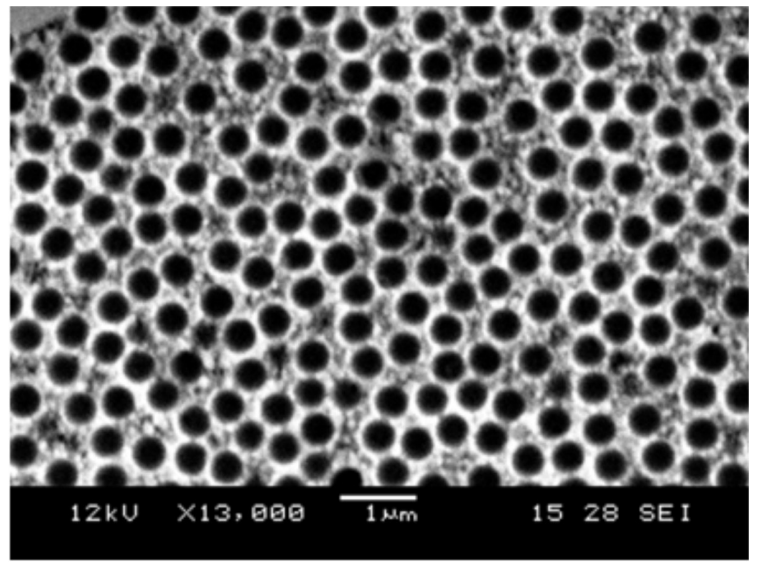

Figura 1. Imagem de microscopia eletrônica de varredura do substrato vidroAu, com a escala indicada na imagem

Observa-se nas imagens a presença de cavidades (círculos escuros) em meio à superfície de Au com diâmetros da ordem de 400 nm, com boa distribuição espacial. As cavidades na superfície são devidas à remoção das microesferas de poliestireno após a eletrodeposição do metal, as quais foram utilizadas como molde para crescimento das estruturas de $\mathrm{Au}$, como indicado pelo esquema da Figura $2 \mathrm{~S}$, material suplementar. Segundo este esquema, nota-se que as dimensões das estruturas de Au dependem da extensão da eletrodeposição e das distâncias entre as microesferas

Uma vez que a imagem da Figura 1 não permite a obtenção de informações referentes à profundidade e, portanto, das dimensões das estruturas de $\mathrm{Au}$ (Figura 2S, material suplementar), foram obtidas imagens das superfícies preparadas através de microscopia de força atômica (AFM), como indicado na Figura 2.

De modo semelhante ao observado nas imagens obtidas por SEM, nota-se, na imagem de AFM, uma boa distribuição espacial das cavidades na superfície de Au. Foi efetuada análise do perfil ao longo da reta indicada na Figura 2, a partir da qual foram definidos os eixos $\mathrm{x} e$ $\mathrm{z}$, como sendo paralelos à reta e normal à superfície, respectivamente. Este perfil é mostrado no inserto à Figura 2, no qual se observa que 


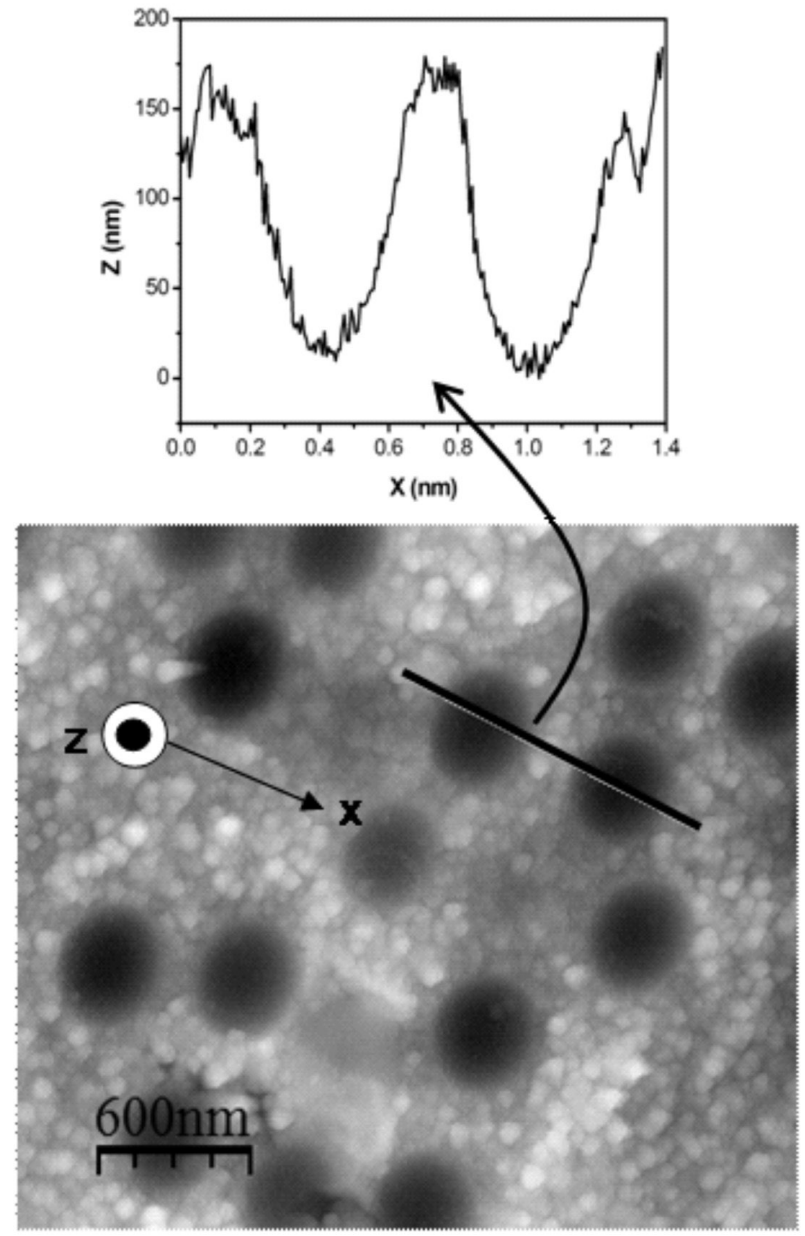

Figura 2. Imagem obtida por microscopia de força atômica do substrato vidro-Au. O perfil de alturas ao longo da reta mostrada na figura é apresentado no inserto

as cavidades apresentam profundidades da ordem de $120 \mathrm{~nm}$ e que, em alguns casos, a separação entre as cavidades é da ordem de 200 nm quando analisado à meia altura das mesmas. Em outros casos, porém, nota-se uma falta de homogeneidade quanto à separação entre as cavidades obtidas, como se pode verificar na própria Figura 2. A caracterização morfológica das nanocavidades demonstra que estas apresentam bordas bem definidas. A presença destas bordas levará a uma alta concentração do campo elétrico local nesta região, como previsto por Mahajan e colaboradores, ${ }^{16} \mathrm{e}$ aumentará o desempenho SERS do substrato.

Na Figura 3S, material suplementar, é apresentado um histograma de distribuição das profundidades de 400 cavidades observadas em imagens obtidas por AFM e na Figura 4S, material suplementar, é apresentado um histograma semelhante para o diâmetro das cavidades. Estes dados se referem, portanto, à distribuição dos tamanhos das estruturas de $\mathrm{Au}$ formadas. $\mathrm{O}$ ajuste de uma distribuição normal permitiu determinar uma média de profundidade em torno de 120 $\mathrm{nm}$, com um desvio da ordem de $60 \mathrm{~nm}$ e a média de diâmetros das nanopartículas em torno de $420 \mathrm{~nm}$, com desvio padrão de $50 \mathrm{~nm}$. Apesar da distribuição relativamente larga dos tamanhos das nanoestruturas obtidas, as mesmas apresentam geometria que permite a intensificação do espectro Raman através da localização do campo elétrico. $^{21}$

Na Figura 3A são apresentados espectros SERS de 4-pyS em concentração de $1,0 \times 10^{-2} \mathrm{~mol} \mathrm{~L}^{-1}$ obtidos em eletrodo de ouro ativado por ciclos de oxidação e redução do metal e no substrato vidro-Au preparado. Estes estão de acordo com os espectros SERS desta molécula reportados na literatura. ${ }^{14,22}$ A comparação entre os espectros SERS obtidos em cada uma das superfícies revela maior eficiência para o substrato vidro-Au em relação ao eletrodo ORC, o que indica boa potencialidade para aplicação como substrato SERS de alto desempenho.
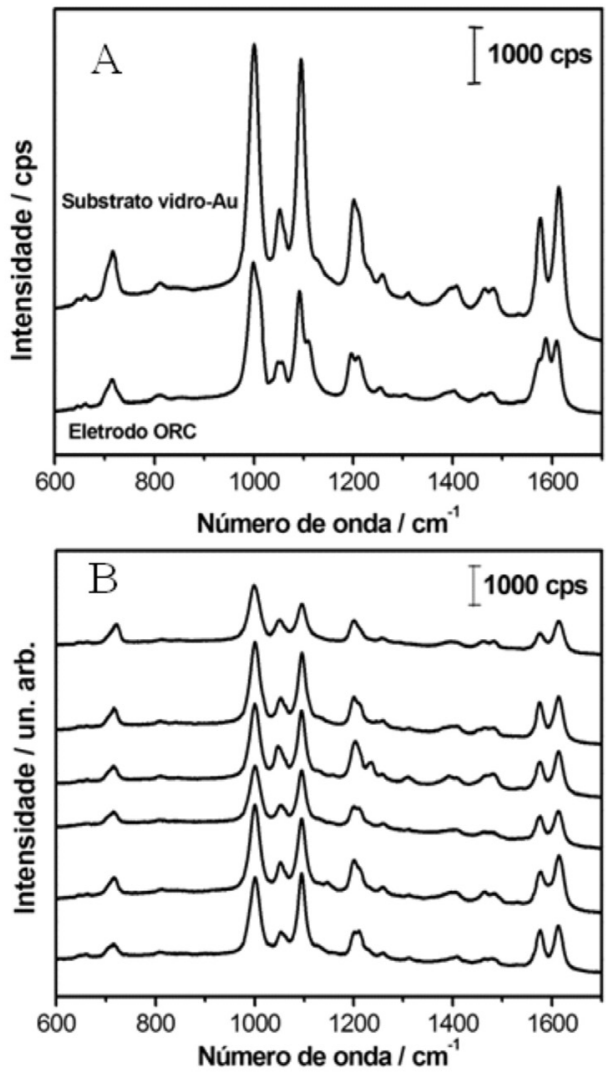

Figura 3. A-Comparação entre os espectros SERS de 4-pyS, 1,0 $010^{-2} \mathrm{~mol}$ $L^{-1}$ no substrato vidro-Au e o eletrodo de Au ativado por ciclos de oxidação e redução. B-Sequência de espectros obtidos em diferentes pontos no substrato vidro-Au

Na Figura 3B são apresentados espectros SERS de 4-pyS nas mesmas condições que na Figura 3A, porém em diferentes regiões da superfície, escolhidos aleatoriamente através do microscópio óptico acoplado ao espectrômetro Raman. Analisando-se os espectros, verifica-se que o sinal SERS obtido nos diversos pontos da superfície apresenta boa reprodutibilidade, no que diz respeito às intensidades relativas das bandas. Além disso, observa-se boa reprodutibilidade em termos das intensidades absolutas. O desvio padrão médio das intensidades SERS da 4-pyS medido nestes substratos vidro-Au foi da ordem de $20 \%$, que é comparável à reprodutibilidade obtida para alguns substratos apresentados na literatura. ${ }^{14,23,24}$

A flutuação nas profundidades e diâmetros das estruturas de Au, observada nas Figuras 3S e 4S (material suplementar), respectivamente, também contribui para uma diminuição na reprodutibilidade do sinal SERS. Esta heterogeneidade leva a uma distribuição de condições de ressonância plasmônica, de tal forma que se tem uma distribuição de intensificações ao longo de toda a superfície, o que leva aos desvios observados de intensidade absoluta das bandas SERS da 4-pyS.

Após verificar a melhor performance do substrato vidro-Au na obtenção dos espectros SERS da 4-pyS, em relação ao eletrodo de 
Au ativado por ORC, foi investigada a eficiência deste substrato para se trabalhar na condição SERS em regime de uma única molécula utilizando a molécula-prova R6G. Na Figura 4A é apresentada uma sequência temporal de espectros SERS de R6G no substrato vidroAu em concentração de $1,0 \times 10^{-9} \mathrm{~mol} \mathrm{~L}^{-1}$. Nessa figura observa-se grande flutuação das bandas nos espectros, com um número alto de eventos nulos, ou seja, de espectros nos quais se observam somente ruído, com alguns espectros de alta intensidade.
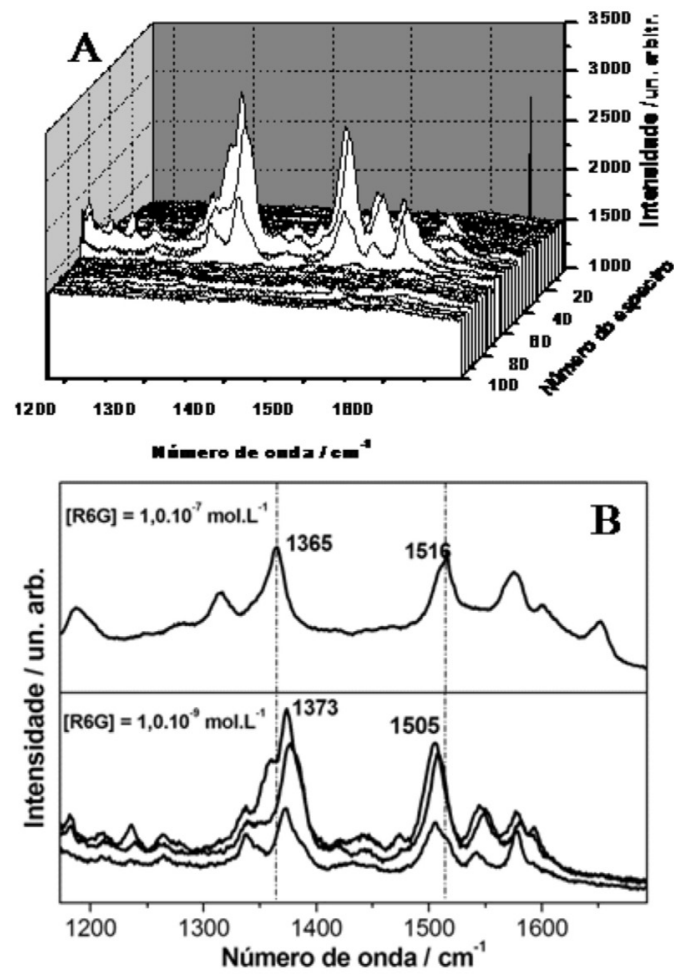

Figura 4. A- Sequência temporal de 100 espectros SERS de R6G 1,0 x 10-9 mol $L^{-1}$ no substrato vidro-Au. B-Comparação entre espectros SERS de alta intensidade mostrados em (A) com espectros SERS de uma solução de R6G, 1,0 $\times 10^{-7} \mathrm{~mol} \mathrm{~L}^{-1}$, nas mesmas condições espectrais

Estes espectros de alta intensidade observados na sequência temporal foram comparados com um espectro SERS de R6G obtido no mesmo substrato, porém em concentrações do analito da ordem de $1 \times 10^{-7} \mathrm{~mol} \mathrm{~L}^{-1}$ (Figura 4B). A comparação na Figura 4B demonstra a boa correlação entre os espectros obtidos em baixa concentração com o espectro da R6G para concentrações altas. Este fato experimental evidencia a possibilidade de utilização do substrato para detecção de concentrações extremamente baixas de analitos através do efeito SERS.

A dinâmica de flutuação espectral observada na Figura 4A é comumente apresentada na literatura como blinking e é normalmente associada ao regime de observação de uma única molécula acessando regiões de altíssimas intensificações na superfície, as quais são normalmente referidas pelo termo hot spots, e que apresentam altas localizações de campo elétrico. ${ }^{25-28}$

Diversos substratos metálicos são apresentados na literatura como sendo capazes de intensificação SERS que permitem a observação de espectros de uma única molécula, como coloides metálicos agregados, ${ }^{29}$ superfícies de Tollens, ${ }^{30}$ entre outros. A obtenção de espectros SERS de uma única molécula tem sido considerada um efeito mais geral, observado mesmo em eletrodos ativados por ciclos de oxidação e redução, e em concentrações não necessariamente tão baixas. ${ }^{31} \mathrm{O}$ substrato vidro-Au apresenta uma maior eficiência na intensificação
SERS em relação ao eletrodo de Au e com maior reprodutibilidade. Este resultado permite inferir que a dinâmica de flutuação espectral observada na Figura 4 é referente a uma ou poucas moléculas adsorvidas em hot spots nestas superfícies.

Superfícies com alto grau de organização, mas com interligações entre as nanoestruturas metálicas permitem dissipação do plasmon de superfície gerado pela interação com a radiação eletromagnética, pela maior contribuição do plasmon de superfície estendido em relação ao localizado. ${ }^{32}$ Desta forma, a localização de campo elétrico nessas estruturas metálicas pode não ser suficiente para a obtenção de intensificações SERS que levem à detecção de uma única molécula. $\mathrm{O}$ substrato vidro-Au utilizado neste estudo apresenta alta interligação entre as estruturas de Au construídas e, portanto, seria esperada uma menor intensificação de campo nestas superfícies, o que não possibilitaria a obtenção de espectros SERS de uma única molécula. Os resultados SERS obtidos sugerem uma causa adicional para a intensificação nestas superfícies que permitiu a localização de campo suficiente para a obtenção de espectros SERS de uma única molécula de R6G.

Wang et al. investigaram diferentes superfícies SERS ativas que possibilitam a obtenção de espectros de uma única molécula através de microscopia de força atômica. ${ }^{33}$ Os autores demonstraram que a raiz quadrática média (RMS - Root Mean Square) da rugosidade da superfície em imagens de AFM com dimensões laterais quadradas $\mathrm{L} \times \mathrm{L}$ apresenta dependência em $\mathrm{L}$ do tipo Lei de Potência, $R M S \propto$ $L^{H}$, até determinadas dimensões de L. Nestes casos, a dimensionalidade fractal, $\mathrm{D}$, foi definida como sendo $\mathrm{D}=3-\mathrm{H}$. As superfícies investigadas apresentaram dimensões fractais de 1,5 para os substratos investigados na ref. 33. A estruturação fractal, ao contrário de superfícies tridimensionalmente organizadas, permite a localização de campo necessária para a observação de espectros SERS de uma única molécula.

Através das imagens de AFM obtidas para os substratos vidro-Au, foram medidos os valores de RMS da superfície para diferentes valores de L. A relação entre o RMS medido com o auxílio do programa WSXM v $5.0^{34}$ e L é apresentada na Figura 5.

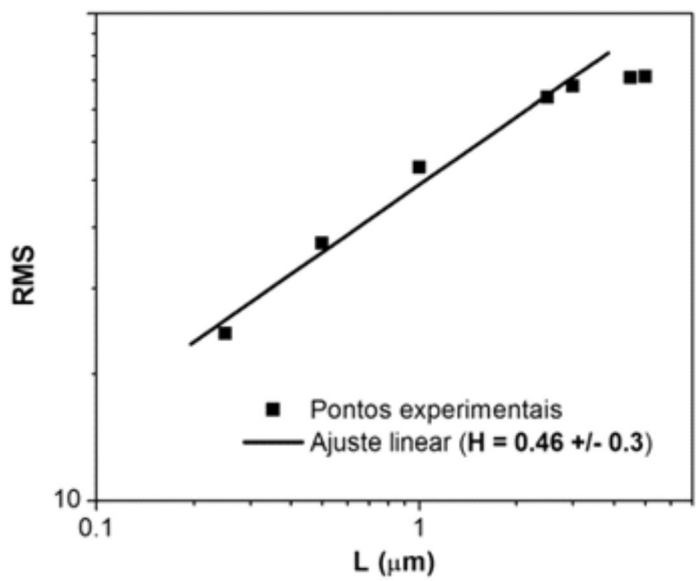

Figura 5. Valor quadrático médio (RMS) em função de L para imagens AFM Lx L do substrato vidro-Au

Na Figura 5 observa-se um comportamento do tipo Lei de potência, em que o coeficiente angular em uma relação do tipo $\log (\mathrm{RMS}) \mathrm{x}$ $\log \mathrm{L}$ corresponde ao valor de $\mathrm{H}$, que neste caso foi observado como sendo de aproximadamente 0,5 . Desta forma, a superfície obtida apresenta uma dimensionalidade fractal de $\mathrm{D}=2,5$ até valores de $\mathrm{L}$ da ordem de 1,5 $\mu \mathrm{m}$. Este resultado justifica a detecção SERS em regime de uma única molécula. A partir de então o valor do coeficiente angular observado é aproximadamente igual a zero, refletindo em uma 
dimensionalidade $\mathrm{D}=3$, não se tratando de uma estruturação fractal.

Para escalas acima de 1,5 $\mu \mathrm{m}$ a organização das cavidades na superfície de Au é o fator mais importante para descrição da estruturação da superfície preparada, como se pode observar na imagem SEM da Figura 1. Ao contrário, porém, a ampliação da resolução permite a visualização de uma rugosidade entre as cavidades obtidas, como se pode observar na imagem AFM da Figura 2, bem como na imagem SEM da Figura 1S, material suplementar. Esta rugosidade, gerada pelo processo de eletrodeposição, é o fator que contribui para a fractalidade da superfície obtida em escala inferior a $1 \mu \mathrm{m}$ e permite a localização do campo elétrico na superfície.

Essas observações explicam as características dos dois substratos comparados no presente trabalho. O eletrodo de Au ativado por ORC apresenta pontos na superfície de alta intensidade SERS, já que possui alta rugosidade superficial e grande dispersão de tamanhos das nanoestruturas presentes, devido ao processo de ativação. Este substrato, porém, apresenta baixa reprodutibilidade nos espectros SERS de uma determinada molécula-prova, devido à alta desorganização das nanoestruturas. A maior reprodutibilidade do substrato vidro-Au deve-se à organização das estruturas entre as cavidades (baixa variação nas formas e tamanhos), enquanto a rugosidade leva à localização de campo na superfície, fator necessário para a obtenção de espectros SERS de uma única molécula nesta superfície.

\section{CONCLUSÕES}

Os resultados obtidos mostraram que o substrato vidro-Au apresenta alta eficiência na obtenção do espectro SERS de adsorbatos moleculares quando comparado com o eletrodo de Au de rugosidade obtida por ciclos de oxidação e redução, com a vantagem de apresentar maior reprodutibilidade espacial entre os espectros obtidos. Além disso, o substrato mostrou-se eficiente na detecção de espectros de soluções de R6G para concentração de até $1,0 \times 10^{-9} \mathrm{~mol} \mathrm{~L}^{-1}$, sendo que a dinâmica espectral observada em uma sequência temporal reflete a dinâmica característica de eventos envolvendo uma única molécula, o que constitui o maior limite de detecção possível para um determinado analito.

\section{MATERIAL SUPLEMENTAR}

Microscopia eletrônica com maior magnificação do substrato vidro-Au (Figura 1S), esquema ilustrando o processo de eletrodeposição em molde de microesferas de PS (Figura 2S), histograma com os valores de profundidade das cavidades, com o valor médio e desvio padrão (Figura $3 \mathrm{~S}$ ) e histograma com os valores dos diâmetros das cavidades, com valor médio e desvio padrão (Figura 4S) encontram-se disponíveis gratuitamente em http://quimicanova.sbq. org.br em arquivo PDF.

\section{AGRADECIMENTOS}

Ao apoio financeiro da FAPESP e do CNPq. D. P. dos Santos e M. L. A. Temperini agradecem ao CNPq pelas bolsas recebidas. G. F. S. Andrade e A. C. Sant'Ana agradecem à FAPESP pelas bolsas recebidas. Os autores agradecem à técnica $\mathrm{A}$. P. Nunes, da central analítica do IQUSP e ao LNLS pelas imagens SEM obtidas, ao técnico M. S. Morlotti pela construção do motor de passos interfaceado, à aluna de mestrado D. C. Ferreira pela obtenção das imagens AFM e ao "Laboratório de Filmes Finos" do Instituto de Física da Universidade de São Paulo pela utilização do equipamento de AFM (FAPESP Proc. No. 95/5651-0).

\section{REFERÊNCIAS}

1. Sala, O.; Fundamentos da Espectroscopia Raman e no Infravermelho, $2^{\text {a }}$ ed., Ed. Unesp: São Paulo, 2008.

2. Aroca, R.; Surface-enhanced vibrational spectroscopy, $1^{\text {st }}$ ed., John Wiley \& Sons: New York, 2006.

3. Kelly, K. L.; Coronado, E.; Zhao, L. L.; Schatz, G. C.; J. Phys. Chem. B 2003, 107, 668 .

4. Le Ru, E. C.; Blackie, E.; Meyer, M.; Etchegoin, P. G.; J. Phys. Chem. C 2007, 111, 13794.

5. Kneipp, K.; Wang, Y.; Kneipp, H.; Perelman, L. T.; Itzkan, I.; Dasari, R. R.; Feld, M. S.; Phys. Rev. Lett. 1997, 78, 1667.

6. Nie, S.; Emory, S. R.; Science 1997, 275, 1102.

7. Otto, A.; J. Raman Spectrosc. 2005, 36, 497.

8. Lombardi, J. R.; Birke, R. L.; Acc. Chem. Res. 2009, 42, 734.

9. Le Ru, E. C.; Etchegoin, P. G.; Principles of Surface-Enhanced Raman Spectroscopy and related plasmonic effects, $1^{\text {st }}$ ed., Elsevier: Amsterdam, 2009.

10. Myroshnychenko, V.; Rodrigues-Fernandez, J.; Pastoriza-Santos, I.; Funston, A. M.; Novo, C.; Mulvaney, P.; Lis-Marzan, L. M.; Abajo, F. J. G.; Chem. Soc. Rev. 2008, 37, 1792.

11. Etchegoin, P. G.; Cohen, L. F.; Hartigan, H.; Brown, R. J. C.; Milton, M. J. T.; Gallop, J. C.; J. Chem. Phys. 2003, 119, 5281.

12. Le Ru, E. C.; Etchegoin, P. G.; Meyer, M.; J. Chem. Phys. 2006, 125, 204701.

13. Natan, M. J.; Faraday Discuss 2006, 132, 321.

14. Dick, L. A.; McFarland, A. D.; Haynes, C. L.; van Duyne, R. P.; J. Phys. Chem. 2002, 106, 853.

15. Batista, E. A.; dos Santos, D. P.; Andrade, G. F. S.; Sant'Ana, A. C.; Brolo, A. G.; Temperini, M. L. A.; J. Nanosci. Nanotechnol. 2009, 9, 3233.

16. Mahajan, S.; Abdelsalam, M.; Suguwara, Y.; Cintra, S.; Russel, A.; Baumberg, J.; Bartlett, P.; Phys. Chem. Chem. Phys. 2007, 9, 104.

17. Olson, L. G.; Lo, Y.; Beebe, T. B.; Harris J. M.; Anal. Chem. 2001, 73, 4268.

18. Oliveira, A. R. M.; Zarbin, A. J. G.; Quim. Nova 2005, 28, 141.

19. Sun, F.; Cai, W.; Li, Y.; Cao, B.; Lu, F.; Guotao, D.; Zhang, L.; Adv. Mater. 2004, 13, 1116.

20. Andrade, G. F. S.; Brolo, A. G.; Temperini, M. L. A.; J. Phys. Chem. C 2008, 112, 15348 .

21. Moskovits, M.; J. Raman Spectrosc. 2005, 36, 485.

22. Chao, Y.; Zhou, Q.; Li, Y.; Yan, Y.; Wu, Y.; J. Chem. Phys. C 2007, 111, 16990.

23. Pesapane, A.; Lucotti, A.; Zerbi, G.; J. Raman Spectrosc. 2010, 41, 256.

24. Fan, M.; Brolo, A. G.; Phys. Chem. Chem. Phys. 2009, 11, 7381

25. Wang, Z.; Rothberg, L. J.; J. Phys. Chem. B 2005, 109, 3387.

26. Pieczonka, N. P. W.; Aroca, R. F.; Chem. Soc. Rev. 2008, 37, 946.

27. Weiss, A.; Haran, G.; J. Phys. Chem. B 2001, 105, 1248.

28. Maher, R. C.; Dalley, M.; Le Ru, E. C.; Etchegoin, P. G.; Hartigan, H.; Brown, R. J. C.; Milton, M. J. T.; J. Chem. Phys. 2004, 121, 8901.

29. Dieringer, J. A.; Lethan II, R. B.; Scheidt, K. A.; van Duyne, R. P.; J. Am. Chem. Soc. 2007, 129, 16249.

30. Shegai, T.; Vaskevich, A.; Rubinstein, I.; Haran, G.; J. Am.Chem. Soc. 2009, 131, 14390 .

31. dos Santos, D. P.; Andrade, G. F. S.; Brolo, A. G.; Temperini, M. L. A.; J. Phys. Chem. C 2009, 113, 17737.

32. Murray, W. A.; Astilean, S.; Barnes, W. L.; Phys. Rev. B 2004, 69, 165407.

33. Wang, Z.; Pan, S.; Krauss, T. D.; Du, H.; Rothberg, L. J.; Proc. Natl. Acad. Sci. USA 2003, 100, 8638.

34. Horcas, I.; Fernández, R.; Gómez-Rodríguez, J. M.; Colchero, J.; Gómez-Herroro, J.; Baro, A. M.; Rev. Sci. Instr. 2007, 78, 13705. 